\title{
Dynamics of xenon binding inside the hydrophobic cavity of pseudo wild-type bacteriophage T4 lysozyme explored through Xenon-based NMR Spectroscopy Supporting Information
}

Hervé Desvaux, ${ }^{1}{ }^{*}$ Lionel Dubois, ${ }^{1}$ Gaspard Huber,${ }^{1}$ Michael L. Quillin, ${ }^{2}$ Patrick Berthault, ${ }^{1}$ and Brian W. Matthews ${ }^{2}$

${ }^{1}$ Laboratoire Structure et Dynamique par Résonance Magnétique, DSM/DRECAM/Service de Chimie Moléculaire, URA CEA/CNRS 331,

C.E.A. Saclay, F-91191 Gif sur Yvette, France. hdesvaux@Cea.fr

${ }^{2}$ Institute of Molecular Biology, Howard Hughes Medical Institute and Department of Physics, 1229 University of Oregon, Eugene, OR 97403-1229, USA.

(Dated: 11th May 2005)

\begin{tabular}{|c|c|c|c|c|c|c|c|}
\hline sidue & Atom & $\Delta \delta_{\max }$ & error & Residue & Atom & $\Delta \delta_{\max }$ & error \\
\hline A74 & $\mathrm{H}^{\mathrm{N}}$ & -0.019 & 0.003 & L121 & $\mathrm{H}_{\alpha}$ & -0.042 & 0.003 \\
\hline V75 & $\mathrm{H}^{\mathrm{N}}$ & -0.028 & 0.002 & L121 & $\operatorname{Me}_{\delta_{1}}$ & 0.072 & 0.002 \\
\hline I78 & $\mathrm{Me}_{\gamma_{2}}$ & -0.014 & 0.002 & L121 & $\mathrm{Me}_{\delta_{2}}$ & -0.158 & 0.002 \\
\hline V87 & $\mathrm{H}^{\mathrm{N}}$ & -0.100 & 0.003 & Q122 & $\mathrm{H}^{\mathrm{N}}$ & -0.032 & 0.002 \\
\hline V87 & $\mathrm{H}_{\beta}$ & -0.068 & 0.002 & E128 & $\mathrm{H}^{\mathrm{N}}$ & -0.028 & 0.005 \\
\hline V87 & $\mathrm{Me}_{\gamma_{1}}$ & -0.029 & 0.004 & A 129 & $\mathrm{H}^{\mathrm{N}}$ & -0.109 & 0.007 \\
\hline Y88 & $\mathrm{H}^{\mathrm{N}}$ & 0.094 & 0.003 & A130 & $\mathrm{H}^{\mathrm{N}}$ & -0.044 & 0.002 \\
\hline D89 & $\mathrm{H}^{\mathrm{N}}$ & -0.020 & 0.003 & V131 & $\mathrm{H}^{\mathrm{N}}$ & 0.067 & 0.002 \\
\hline 992 & $\mathrm{H}^{\mathrm{N}}$ & 0.024 & 0.001 & N132 & $\mathrm{H}^{\mathrm{N}}$ & 0.037 & 0.003 \\
\hline A93 & $\mathrm{H}^{\mathrm{N}}$ & -0.029 & 0.003 & L133 & $\mathrm{H}^{\mathrm{N}}$ & 0.074 & 0.002 \\
\hline V94 & $\mathrm{H}^{\mathrm{N}}$ & -0.025 & 0.004 & L133 & $\mathrm{Me}_{\delta_{1}}$ & -0.104 & 0.003 \\
\hline F104 & $\mathrm{H}^{\mathrm{N}}$ & -0.038 & 0.003 & A134 & $\mathrm{H}^{\mathrm{N}}$ & 0.119 & 0.002 \\
\hline F104 & $\mathrm{H}_{\epsilon}$ & 0.034 & 0.001 & A134 & $\mathrm{H}_{\beta}$ & 0.053 & 0.003 \\
\hline V111 & $\mathrm{H}_{\alpha}$ & 0.050 & 0.002 & W138 & $\mathrm{H}_{\alpha}$ & -0.043 & 0.003 \\
\hline 114 & $\mathrm{H}^{\mathrm{N}}$ & -0.065 & 0.003 & W138 & $\mathrm{H}_{\epsilon}$ & 0.041 & 0.003 \\
\hline $\mathrm{F} 114$ & $\mathrm{H}_{\alpha}$ & -0.041 & 0.005 & Y139 & $\mathrm{H}_{\alpha}$ & -0.030 & 0.003 \\
\hline F114 & $\mathrm{H}_{\beta_{2}}$ & 0.060 & 0.002 & Q141 & $\mathrm{H}^{\mathrm{N}}$ & -0.050 & 0.002 \\
\hline 114 & $\mathrm{H}_{\delta}$ & 0.059 & 0.004 & T142 & $\mathrm{H}^{\mathrm{N}}$ & -0.089 & 0.003 \\
\hline F114 & $\mathrm{H}_{\epsilon}$ & -0.034 & 0.002 & T142 & $\mathrm{H}_{\alpha}$ & -0.026 & 0.002 \\
\hline F114 & $\mathrm{H}_{\zeta}$ & -0.034 & 0.004 & T142 & $\mathrm{Me}_{\gamma}$ & 0.106 & 0.002 \\
\hline Г115 & $\mathrm{H}^{\mathrm{N}}$ & 0.058 & 0.001 & V149 & $\mathrm{Me}_{\gamma_{1}}$ & 0.032 & 0.003 \\
\hline L118 & $\mathrm{Me}_{\delta_{2}}$ & 0.101 & 0.001 & I150 & $\mathrm{H}^{\mathrm{N}}$ & 0.037 & 0.004 \\
\hline 119 & $\mathrm{H}^{\mathrm{N}}$ & -0.128 & 0.004 & T151 & $\mathrm{H}^{\mathrm{N}}$ & -0.044 & 0.002 \\
\hline M120 & $\mathrm{H}^{\mathrm{N}}$ & 0.128 & 0.003 & T157 & $\mathrm{H}^{\mathrm{N}}$ & -0.034 & 0.001 \\
\hline & & 0.08 & & & & & \\
\hline
\end{tabular}

Table I: List of the assigned protons experiencing specific chemical shift variation under xenon pressure. The value $\Delta \delta_{\max }$ is computed for $K=60.2 \mathrm{M}^{-1}$.

\footnotetext{
${ }^{*}$ Corresponding author
} 


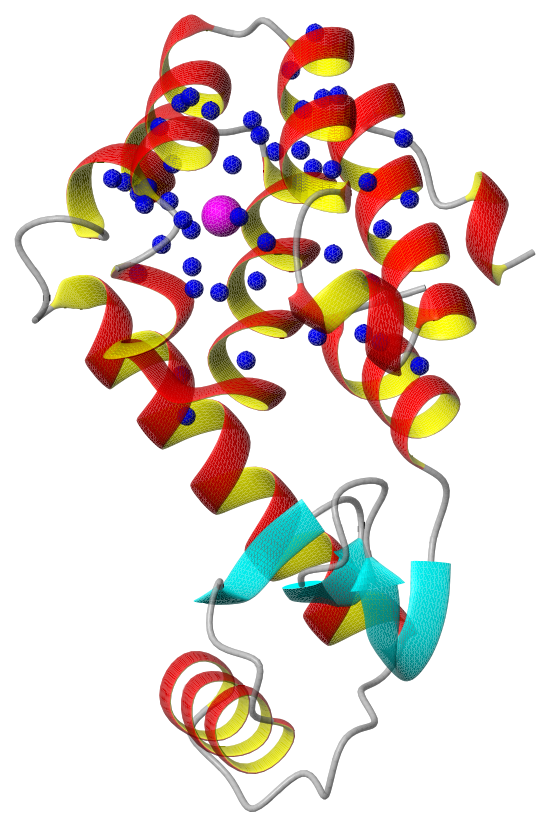

Figure 1: Structure of T4 lysozyme (PDB code: 1C6T). The ${ }^{1} \mathrm{H}$ atoms exhibiting a chemical shift variation under xenon pressure are represented by spheres.

\begin{tabular}{|c|c|c|c|}
\hline Residue & Atom & Residue & Atom \\
\hline I78 & $\mathrm{H}_{\alpha}$ & L118 & $\mathrm{H}_{\beta}$ \\
\hline I78 & $\mathrm{Me}_{\gamma_{2}}$ & L118 & $\mathrm{Me}_{\delta_{1}}$ \\
\hline V87 & $\mathrm{Me}_{\gamma_{1}}$ & L118 & $\mathrm{Me}_{\delta_{2}}$ \\
\hline V87 & $\mathrm{Me}_{\gamma_{2}}$ & L121 & $\mathrm{H}_{\gamma}$ \\
\hline Y88 & $\mathrm{H}_{\delta}$ & L121 & $\mathrm{Me}_{\delta_{1}}$ \\
\hline Y88 & $\mathrm{H}_{\epsilon}$ & L133 & $\mathrm{Me}_{\delta_{1}}$ \\
\hline V103 & $\mathrm{H}_{\alpha}$ & L133 & $\mathrm{Me}_{\delta_{2}}$ \\
\hline V103 & $\mathrm{Me}_{\gamma_{1}}$ & V149 & $\mathrm{H}_{\beta}$ \\
\hline V111 & $\mathrm{Me}_{\gamma_{2}}$ & F153 & $\mathrm{H}_{\zeta}$ \\
\hline
\end{tabular}

Table II: List of the protons detected in the SPINOE spectra. 


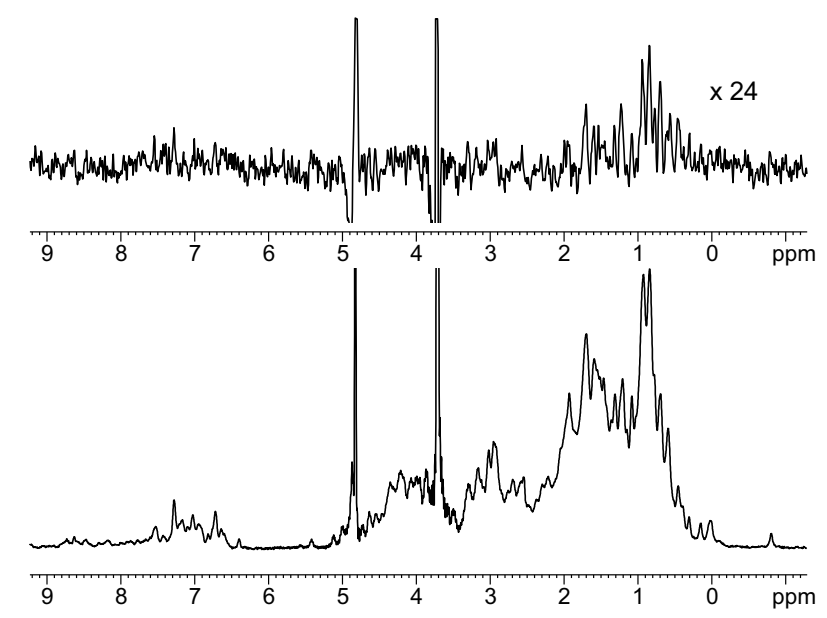

Figure 2: ${ }^{1} \mathrm{H}$ NMR spectrum (bottom) and SPINOE spectrum (top) of the WT* T4 lysozyme protein acquired at $500 \mathrm{MHz}$. The mixing time is equal to $600 \mathrm{~ms}$. The spectrum corresponds to a composition of nine different experiments acquired in three different runs (new batches of laser-polarized xenon).

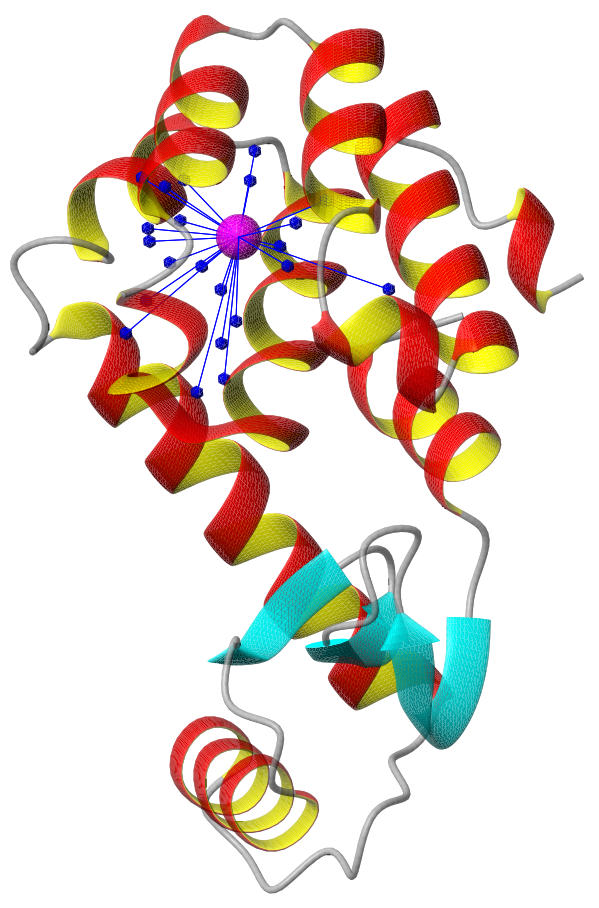

Figure 3: Structure of T4 lysozyme (PDB code: $1 \mathrm{C} 6 \mathrm{~T})$. The ${ }^{1} \mathrm{H}$ atoms detected by SPINOE are represented by spheres. The lines correlate the xenon atom, as determined by X-ray crystallography, and the detected protein protons. 